\title{
DTI-DROID: Diffusion Tensor Imaging-Deformable Registration Using Orientation and Intensity Descriptors
}

\author{
Madhura Ingalhalikar, Jinzhong Yang, Christos Davatzikos, Ragini Verma \\ Department of Radiology, Section of Biomedical Image Analysis, University of Pennsylvania, \\ PA 19104
}

Received 2 April 2010; accepted 2 April 2010

\begin{abstract}
This article presents a method (DROID) for deformable registration of diffusion tensor (DT) images that utilizes the full tensor information by integrating the intensity and orientation features into a hierarchical matching framework. The intensity features are derived from eigen value based measures that characterize the tensor in terms of its different shape properties, such as, prolateness, oblateness, and sphericity of the tensor. Local spatial distributions of the prolate, oblate, and spherical geometry are used to create an attribute vector called the geometric/intensity feature for matching. The orientation features are the orientation histograms computed from the eigenvectors. These intensity and orientation features are incorporated into a hierarchical deformable registration framework to develop a deformable registration algorithm for DT images. Using orientation features improves the matching of the white matter fiber tracts by taking into account the underlying fiber orientation information. Extensive experiments on simulated and real brain DT data show promising results that makes DROID potentially useful for subsequent group-based analysis of DT images to identify disease-induced and developmental changes in a population. (c) 2010 Wiley Periodicals, Inc. Int J Imaging Syst Technol, 20, 99-107, 2010; Published online in Wiley InterScience (www.interscience. wiley.com). DOI 10.1002/ima.20232
\end{abstract}

Key words: diffusion tensor; deformable registration; intensity descriptors; orientation descriptors

\section{INTRODUCTION}

Diffusion tensor imaging (DTI) has emerged as a powerful and effective technique for analyzing the underlying white matter (WM) structure of brains (Pierpaoli et al., 1996). DTI provides unique micro-structural and physiological insight into WM tissue microstructure of brains, which in turn facilitates the study of development, aging, and disease on specific WM regions of interest. To carry out group-based analysis and statistics, it is imperative to make different subjects comparable, thus requiring the spatial normalization of diffusion tensor (DT) images. However, spatial normalization of DT images is rendered challenging by the fact that the data representation is high dimensional and tensors have an orientation component. This requires spatial warping, followed by ten-

Correspondence to: Ragini Verma; e-mail: ragini.verma@uphs.upenn.edu Grant support: NIH R01MH079938, T32-EB000814, R01MH060722 sor reorientation at each voxel (Alexander et al., 2001; Xu et al., 2003).

Registration methods for DTI can be categorized on the information that they use for matching the two images (establishing correspondence), that is, the full tensor (Zhang et al., 2006; Yang et al., 2008) or scalar measures (Xu et al., 2003) and at what stage they perform tensor reorientation. Early methods in DTI registration used scalar measures such as fractional anisotropy (FA) to align the data. More recently, a combination of different scalar maps derived from full tensor image are used for a multi-channel DT image registration (Guimond et al., 2002). These methods do not incorporate orientation information from the tensors (as only eigen value-based measures are used) and hence are not expected to align WM fibers completely. To fully utilize the information provided by DT images, more advanced algorithms were proposed. In Cao et al. (2006), Zhang et al. (2006), Yeo et al. (2008), instead of the scalars derived from DT image, the full tensor similarity measurements is adopted. Using tensors, utilizes the important orientation information, however makes it difficult to incorporate spatial neighborhood information, important in registration.

Irrespective of the information used for matching, DTI registration methods also differ on the basis of at what stage tensor reorientation is performed. In Alexander and Gee (1999), Alexander (2001), Shen et al. (2002), tensor reorientation is not taken into account in the objective function when solving the deformable registration problem. The reorientation is computed from the deformation field obtained from the spatial warping step, using one of the two commonly used reorientation strategies: the finite-strain and the preservation of principal directions (PDs) (Alexander et al., 2001). This is perfectly justified when rotation invariant features are used for matching, such as FA-based methods, however, when tensor information is used for matching as in Zhang et al. (2006), reorientation needs to be performed in each step of the optimization process. This is done by algorithms (Cao et al., 2006; Zhang et al., 2006; Yeo et al., 2008), which compute the exact gradient of the tensor reorientation during optimization. While, using full tensor information for registration or taking reorientation into account when computing the gradient of the objective function provides an improvement over the registration provided by scalar measures, it is 
difficult to incorporate neighborhood information, thereby limiting the matches to be very local, subsequently making it prone to local minima issues. Also incorporating reorientation information at each step of the optimization increases the computational load.

The issue of incorporating neighborhood information with orientation-based features has led to the development of feature-based algorithms in which features extracted from the tensors are used for registration. While these have been used for registration of structural images (Shen and Davatzikos, 2002) they have not been extensively researched for tensors. An earlier study applied oriented 3D Gabor features extracted from tensors for matching (Verma et al., 2004), while a recent method employed major fiber bundles to align tensor (Ziyan et al., 2007). Both methods demonstrate that registration can be improved in the WM if features that characterize both tensor geometry and orientation are used for matching with carefully chosen metrics.

In this article, we use tensor shape and orientation features for DTI registration. We capitalize on the structural geometry of DT (Westin et al., 2002) and develop a novel attribute vector consisting of geometric moments computed from the local spatial histograms of tensor geometric measures. This attribute vector is rotationally invariant, and integrates spatial information from local histograms computed at different scales. To improve the registration accuracy of WM fiber tracts, we also incorporate the local information of underlying fiber orientations for feature matching. These features provide richer anatomical information from the tensor by integrating anisotropy, shape, and orientation from the entire neighborhood of a voxel. We include these features into a hierarchical deformable registration technique, to develop a deformable registration method for DT images. As the method used both intensity and orientation features, it is called deformable registration using orientation and intensity descriptors (DROID). While shape features are adequate in performing a good registration between images, the orientation features serve to align the WM tracts better. Extensive experiments demonstrate the robustness and accuracy of DT image registration using these features. Results obtained from our algorithm have been compared with other deformable registration algorithms.

\section{REGISTRATION METHOD}

We propose a feature-based deformable registration algorithm for DTI data. We define features (or attribute vectors) at each voxel of the image to determine the matching pairs of points during the image registration procedure. In our method, we use a combination of intensity (geometry) and orientation histograms, computed at each voxel, from multi-resolution images. Moreover, we use edge information also extracted from the image for discriminating boundary points between different tissue types, from others. The attribute vectors are described in section A. Section B describes the registration algorithm.

A. Creation of Attribute Vectors from Tensors. A tensor provides information about the magnitude and orientation of water diffusivity at any given voxel. A tensor $\mathbf{D}$ can be decomposed into

$$
\mathbf{D}=\lambda_{1} \hat{\mathbf{e}}_{1} \hat{\mathbf{e}}_{1}^{T}+\lambda_{2} \hat{\mathbf{e}}_{2} \hat{\mathbf{e}}_{2}^{T}+\lambda_{3} \hat{\mathbf{e}}_{3} \hat{\mathbf{e}}_{3}^{T}
$$

where $\lambda_{1} \geq \lambda_{2} \geq \lambda_{3} \geq 0$ are the three eigen values of a symmetric tensor $\mathbf{D}$, which characterize the magnitude of water diffusivity and $\hat{\mathbf{e}}_{i}$ is the normalized eigenvector corresponding to $\lambda_{i}$, which refers to the direction of water diffusivity. Geometrically, tensor $\mathbf{D}$ is represented by an ellipsoid with three axes oriented along its three eigenvectors, and three semi-axis lengths proportional to the square root of its three eigen values. The eigen values, therefore, characterize the shape or geometry of the tensors and the eigenvectors characterize the orientation. Different shapes of the ellipsoid give rise to three geometric structures of DTs: prolate (linear) structure $\left(C_{1}\right)$, in which diffusion is mainly in the direction corresponding to $\hat{\mathbf{e}}_{1}$ (the principal diffusivity); oblate (planar) structure $\left(C_{\mathrm{p}}\right)$, in which diffusion is restricted to a plane spanned by $\hat{\mathbf{e}}_{1}$ and $\hat{\mathbf{e}}_{2}$; and spherical structure $\left(C_{\mathrm{s}}\right)$ with isotropic diffusion. Three geometric measures were proposed in Westin and Maier (2002) to describe how close the DT is to the generic structures of prolateness, oblateness, and sphericity. Mathematically, they are defined as

$$
c_{1}=\frac{\lambda_{1}-\lambda_{2}}{\lambda_{1}}, c_{\mathrm{p}}=\frac{\lambda_{2}-\lambda_{3}}{\lambda_{1}}, c_{\mathrm{s}}=\frac{\lambda_{3}}{\lambda_{1}}
$$

We use these shape features to define intensity attributes and eigen vectors to determine orientation features.

A.1. Intensity Descriptors: Tensor Geometric Features. A discriminative attribute vector is defined at each voxel from the geometric measures in Eq. (2). This attribute vector characterizes the local diffusion property by combining the local distributions of prolate, oblate, and spherical structures. For a specified voxel $v$, local intensity histograms $\mathbf{h}_{\mathrm{l}}(v)$ of $c_{\mathrm{l}}, \mathbf{h}_{\mathrm{p}}(v)$ of $c_{\mathrm{p}}$, and $\mathbf{h}_{\mathrm{s}}(v)$ of $c_{\mathrm{s}}$ are computed from a spherical neighborhood region of voxel $v$ with an appropriate radius $r$. These histograms roughly characterize the distribution of the tensor geometry in the neighborhood region. For each histogram, we compute its regular geometric moments as the statistical geometric features that characterize the shape, i.e.,

$$
m_{k}(v, n)=\sum_{i} i^{n} \mathbf{h}_{k}(v, i), \quad k=1, \mathrm{p}, \mathrm{s}
$$

where $\mathbf{h}_{k}(v, i)$ is the frequency of index $i$ in histogram $\mathbf{h}_{k}(v)$, and $m_{k}(v, n)$ is the $n$th order moment of this histogram. Low order geometric moments are used to represent the local geometric features for a histogram and form a vector as

$$
\mathbf{a}_{k}^{\text {hist }}(v)=\left\{m_{k}(v, n) \mid n=0,1,2\right\}, \quad k=1, \mathrm{p}, \mathrm{s} .
$$

The higher order moments provide more global information. To improve the accuracy of matching, we include the edge strength $b_{\mathrm{FA}}^{\text {edge }}(v)$ of FA and the edge strength $b_{\mathrm{ADC}}^{\text {edge }}(v)$ of apparent diffusion coefficient (ADC) into the attribute vector. These edge attributes are computed by a Canny edge detector (Canny, 1986) from FA and ADC scalar maps of DT image, respectively. Therefore, the complete attribute vector at voxel $v$ can be represented as

$$
\mathbf{a}(v)=\left[\mathbf{a}_{1}^{\text {hist }}(v), \mathbf{a}_{\mathrm{p}}^{\text {hist }}(v), \mathbf{a}_{\mathrm{s}}^{\text {hist }}(v), b_{\mathrm{FA}}^{\text {edge }}(v), b_{\mathrm{ADC}}^{\text {edge }}(v)\right] .
$$

The attribute vector defined in Eq. (5) is rotationally invariant, which makes it attractive for registration. To make the feature vector more discriminative, the above attribute vector is computed at three different scales so that both global and local geometric features are accounted for. In each scale, the similarity of two attribute vectors, $\mathbf{a}(u)$ and $\mathbf{a}(v)$, of two points, $u$ and $v$, is defined as

$$
m(\mathbf{a}(u), \mathbf{a}(v))=\prod_{i}\left(1-\left|\mathbf{a}_{i}(u)-\mathbf{a}_{i}(v)\right|\right),
$$

where $\mathbf{a}_{i}(\cdot)$ is the $i$ th element in the attribute vector. As $c_{1}, c_{\mathrm{p}}$, and $c_{\mathrm{s}}$ add up to one, information from all three may be overlapping, 
therefore we discard the $0^{\text {th }}$ and $1^{\text {st }}$ order geometric moments derived from $c_{\mathrm{p}}$ when computing the similarity in Eq. (6).

A.2. Orientation Descriptors of Tensors. Proper alignment of WM fiber tracts is a major concern in DTI registration. To further improve the registration accuracy of WM fiber tracts, we incorporate the local statistical information of underlying fiber orientations into the attribute vector defined in Eq. (5). The fiber orientation at voxel $v$ is approximated by the PD of tensor $\mathbf{D}$ weighted by the FA value at this voxel. Local spatial distribution of PD in the $3 \mathrm{D}$ space at voxel $v$, denoted by $\mathbf{H}_{\mathrm{PD}}(v)$, can be estimated from the samples in a spherical neighborhood region with a radius $r$. The similarity of two points, $u$ and $v$, in terms of local PD distribution is characterized by entropy cross correlation (Huang and Sun, 2004), a normalized form of mutual information, as

$$
\operatorname{ECC}_{\mathrm{PD}}(u, v)=2-2 \frac{E\left[\mathbf{H}_{\mathrm{PD}}(u), \mathbf{H}_{\mathrm{PD}}(v)\right]}{E\left[\mathbf{H}_{\mathrm{PD}}(u)\right]+E\left[\mathbf{H}_{\mathrm{PD}}(v)\right]},
$$

where $E$ denotes the joint or marginal differential entropy of the random variables of local PD distribution. The similarity with the orientation features at points $u$ and $v$ is then determined by the combination of Eqs. (6) and (7) as

$$
M(u, v)=m(\mathbf{a}(u), \mathbf{a}(v)) \cdot \operatorname{ECC}_{\mathrm{PD}}(u, v),
$$

$M(u, v)$ ranges from zero to one where one indicates the most similar features. As the PD of a tensor is meaningful only in high anisotropic anatomies such as WM fiber tracts, we consider the matching of orientation feature only for voxels with FA value higher than 0.5 .

\section{B. Deformable Registration with Orientation and Intensity}

Descriptors. As a first step, the two images are aligned linearly using FLIRT (Jenkinson and Smith, 2001) on the corresponding FA images. To develop a DTI registration algorithm, the attribute vectors of tensor intensity and orientation features described above are used in conjunction with the energy function of the intensity histogram-based HAMMER algorithm (Shen 2007). Attribute vectors are computed for both the subject and the template image.

Let $\mathrm{a}_{\mathrm{T}}(\mathrm{u})$ denote the attribute vector of a point $u$ in the template image $T(u)$, and let $\mathrm{a}_{\mathrm{S}}(\mathrm{v})$ represent the attribute vector of a point $v$ in the subject image $S(v)$. The template image $T(u)$ is deformed to match with the subject $S(v)$ by a displacement field $d(u)$, or equally a forward transformation $h(u)$. The backward transformation from the subject to the model is $h^{-1}(u)$, which is the inverse of the forward transformation $h(u)$. The following is the function [Eq. (9)] that will be minimized as part of the registration algorithm:

$$
\begin{aligned}
& F=\sum_{u} w_{T}(u)\left\{\frac{\sum_{z \in n(u)} \epsilon(z)\left(1-m\left(a_{T}(z), a_{S}(h(z))\right)\right)}{\sum_{z \in n(u)} \epsilon(z)}\right\}+ \\
& \sum_{v} w_{S}(v)\left\{\frac{\sum_{z \in n(v)} \epsilon(z)\left(1-m\left(a_{T}\left(h^{-1}(z)\right), a_{S}(z)\right)\right)}{\sum_{z \in n(v)} \epsilon(z)}\right\}+\beta \sum_{u}\left\|\nabla^{2} d(u)\right\|
\end{aligned}
$$

There are three terms in this function: first term evaluates the match of template with subject, by using forward transformation $h($.); the second energy term evaluates the match of subject with template, by using backward transformation $h^{-1}($.). This ensures inverse consistency, that is, the transformation between the subject and the template is identical, irrespective of which of the two is used as a template (Christensen and Johnson, 2001), and the third term ensures the smoothness of the deformation fields. The first energy term is defined as the weighted summation of match of all points in the neighborhood of point $u$ in the template image. $\mathrm{W}_{\mathrm{T}}(\mathrm{u})$ is used as a weight for the point $u$, which can be adaptively adjusted by boundary attributes during the image registration procedure. This allows the hierarchical selection of active points, thus enabling the approximation of a high dimensional cost function (equal to the number of points in the two images) by a significantly low dimensional function of only the active points. The active points used to drive the algorithm are selected according to the edge map of FA and ADC maps. To reduce the ambiguity in finding correspondences, a hierarchical strategy of active points selection is employed. In the initial stages of the matching procedure, only a few points with high Canny edge strengths are selected for matching to avoid local minima. As the matching progresses, more and more points with lower strength become reliable and thus are selected to drive the registration. This function based on active points has fewer local minima, because it is a function of the coordinates of active points, for which relatively unambiguous matches can be found. For a point $u$, the degree of its neighborhood match is defined as the similarity of all attribute vectors in the neighborhood, $n(u)$. This design thereby allows the neighborhood matching during the image registration, which effectively increases the robustness to potentially false matches of active points. Here, $z$ is a neighboring point of $u$, and its attribute vector $\mathrm{a}_{\mathrm{T}}(\mathrm{z})$ is compared with the attribute vector $\mathrm{a}_{\mathrm{S}}(\mathrm{h}(\mathrm{z}))$ of its corresponding point $h(z)$ in the subject; the similarity is defined as $\overline{\mathrm{d}}($,$) , thereby the difference is 1-\overline{\mathrm{d}}($,$) . The term in the$ denominator is used for normalization. The design of the second energy term is the same as the first with the transformation $h^{-1}($.). The third energy term is used to make sure that the resulting displacement fields are smooth, by requiring the total Laplacian value of displacement fields to be as small as possible. The parameter $\beta$ controls the smoothness of the deformation fields.

To speed up the algorithm, as a first step, only the geometry/ intensity feature [as shown in Eq. (5)] is used for registration. These features are extracted once and not recalculated during registration iterations. As these features are rotationally invariant, no explicit reorientation is required in each step of the optimization. The deformation field obtained as part of the spatial warping is used to determine the final tensor reorientation, based on a spatially adaptive procedure that estimates the underlying fiber orientation (Xu et al., 2003), to produce properly reoriented tensors. Next, we further refine the matching of WM fiber tracts by using orientation features, in the second stage of registration. As the orientation information is meaningful only at anisotropic voxels, orientation features are computed only at the voxels with high anisotropy. As orientation features are not rotationally invariant, they are extracted in each iteration and the tensors are warped and reoriented accordingly. The final spatial transformation is generated by concatenating the hierarchical sequence of piecewise smooth transformations obtained at each stage (without and with orientation feature). Tensor reorientation is performed a second time, after the matching based on orientation features. As the images are quite well registered after the first stage of shapebased registration, reorientation can be maintained as an outside step of the optimization, to lower computation costs. 


\section{DTI Datasets and Evaluation of Registration.}

C.1. DTI Acquisition and Preprocessing. The first dataset used for validation of DROID was a simulated dataset. Ten nonlinear deformation fields were generated using Xue et al. (2006) in which the atrophy was introduced in different tissues. These simulated fields were applied to a DT image chosen as the template, to generate 10 simulated brains.

In the second dataset, images were acquired using Siemens 3T Tim Trio Scanner. Each dataset consisted of 64 gradient directions with the diffusion weighting of $b=1000 \mathrm{~s} / \mathrm{mm}^{2}(\mathrm{NEX}=2)$ and six nonweighted images. The DTI data were reconstructed using FSL tools (Smith et al., 2004; Xue et al., 2006) in which a tensor was fitted at each voxel. The reconstructed DTI data had the data resolution as $1.73 \times 1.73 \times 2 \mathrm{~mm}$.

For computing the brain atlas, two different datasets were used. First one was ex vivo mouse data, acquired on a 9.4 T Bruker scanner equipped with triple-axis gradients, and Bruker commercial volume coils (10-25 mm inner diameter) as dual purpose radio-frequency transmitter and receiver. The data was acquired with six independent diffusion weighted directions with $b$ value $=1000 \mathrm{~s} /$ $\mathrm{mm}^{2}$, two additional images with minimal diffusion weighting $(b=$ $50 \mathrm{~s} / \mathrm{mm}^{2}$ ). Six repetitions were acquired. The reconstructed image resolutions ranged from $62.5 \times 100 \times 100 \mu \mathrm{m}$ to $125 \times 125 \times 125$ $\mu \mathrm{m}$. The second dataset consisted of 30 human brain scans and was acquired using Philips 1.5 T REC Scanner. Thirty gradient directions were used with the diffusion weighting of $b=700 \mathrm{~s} / \mathrm{mm}^{2}$ $(\mathrm{NEX}=2)$. The DTI data were reconstructed using FSL tools (Smith et al., 2004) in which a tensor was fitted at each voxel. The reconstructed DTI data had the data resolution of $0.9375 \times 0.9375$ $\times 2.5 \mathrm{~mm}$. All the datasets were obtained in compliance with the Institutional Review Board.

C.2. Registration Experiments and its Evaluation. Evaluating registration is challenging as there is no gold standard to serve a baseline. A commonly used approach is to apply the registration algorithm, in our case DROID on a simulated dataset and check if it produced the original deformation field. On the real datasets, the evaluation was based on different criteria. First, a manual landmark matching was performed by two different raters. Also, a comparison with other deformable registration techniques was performed and FA variance and tensor overlap was computed.

C.2.1. Validation Based on Simulated Data. In this experiment, we evaluate the registration accuracy against a known deformation introduced in the brain. The aim is to determine how well the registration algorithm is able to obtain the simulated deformation that has been added to the brain image. Ten simulated DT images (described in the Methods subsection C.1) were registered to the template space by DROID. For testing the similarity between the deformed tensor and the template tensor at each voxel, we used overlap index (OVL) criterion (Basser and Pajevic, 2000). If the tensors overlap perfectly the OVL value is one while for tensors with low overlap OVL is close to zero. The overlap index is given by Eq. (10), where $\lambda_{i}$ are the eigen values and $e_{i}$ are the eigenvectors of one tensor while $\lambda_{i}^{\prime}$ and $e_{i}^{\prime}$ are the eigen values and eigenvectors of the other tensor.

$$
\mathrm{OVL}=\frac{\sum_{i=1}^{3} \lambda_{i} \lambda_{i}^{\prime}\left(e_{i} \cdot e_{i}^{\prime}\right)^{2}}{\sum_{i=1}^{3} \lambda_{i} \lambda_{i}^{\prime}}
$$

Evaluation was also based on comparing with a known FA-based registration implemented in $\mathrm{Xu}$ et al. (2003). In the latter case, only FA information was used to generate the feature, as compared to our algorithm in which several anisotropy and diffusivity measures are used to create the feature. We applied our method using just the intensity features for a close comparison against the FA-based registration. The vector-wise difference between the two deformation fields produced by the registration of the simulated brains to the template and the simulated ground truth were calculated for both methods.

C.2.2. Validation of DROID on Real Data. After any registration it is important that each anatomical landmark matches exactly with the template image. Therefore, we compared the ability of our method in identifying point matches, against human raters. We then compared its performance with the Demons algorithm applied to the FA images. The demons registration was taken from ITK (www.itk.org) and was performed with initial histogram matching and using three levels of refinement with a smoothing sigma factor

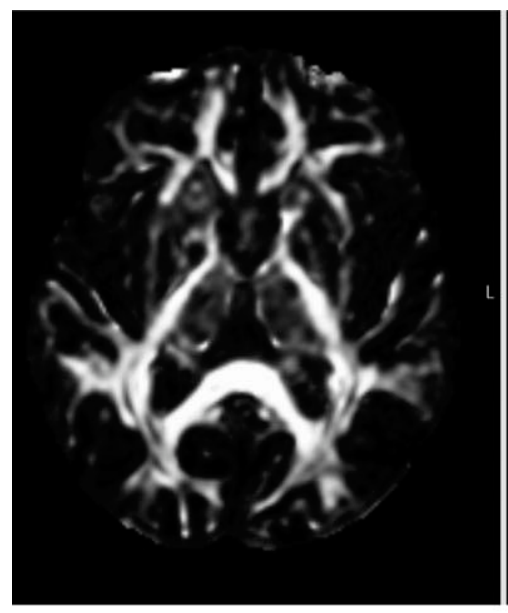

(a)

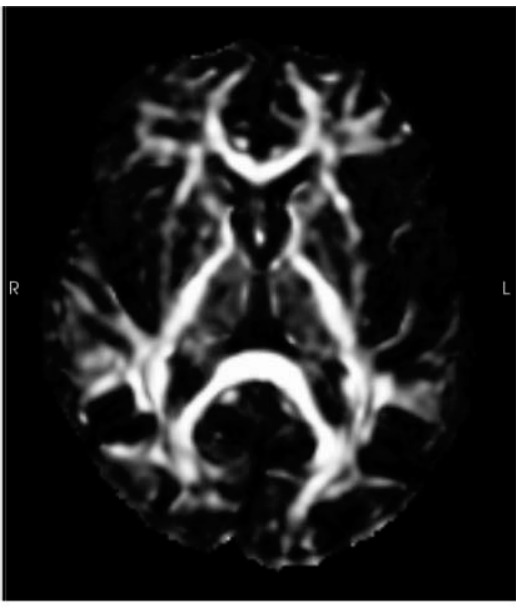

(b)

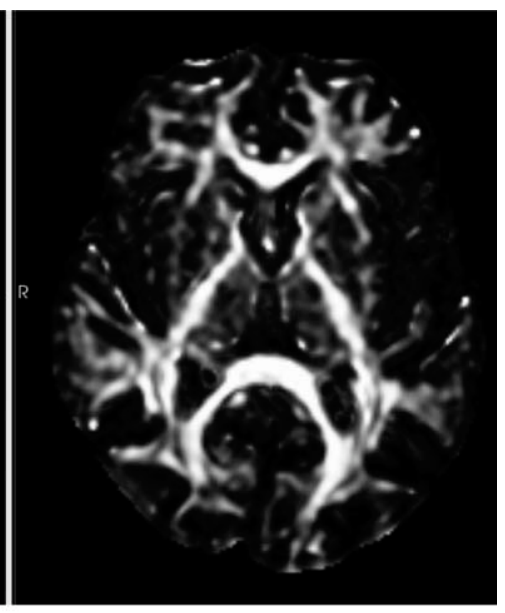

(c)

Figure 1. Figure shows the results of DROID on the nonlinearly simulated datasets. (a) One of the simulated dataset that was used as a moving image while, (b) the deformed image after registration, (c) the template image. The tensor images have been converted to scalar FA images for visualization. 


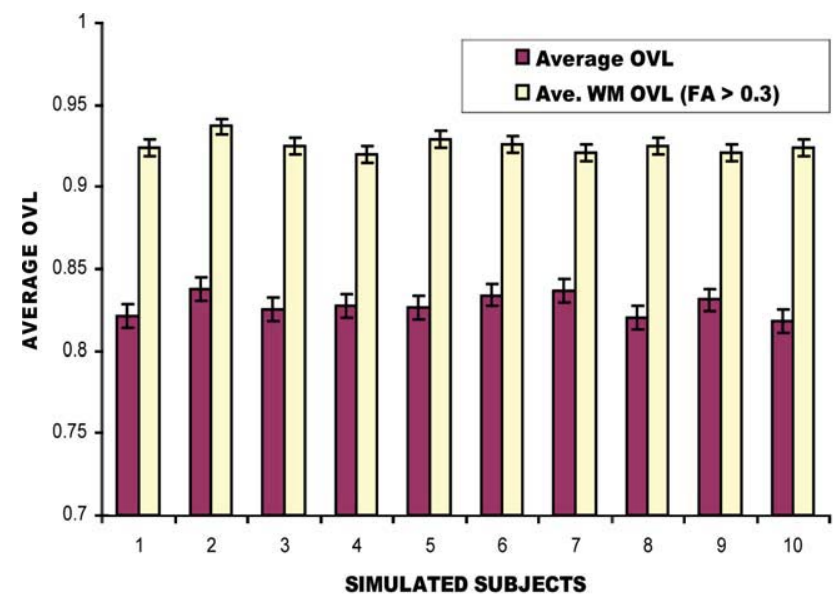

(a)

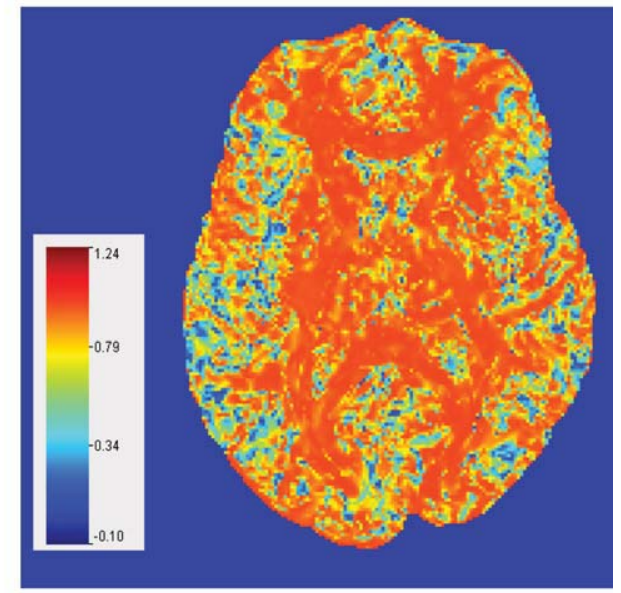

(b)

Figure 2. The plot shows the tensor overlap for all the 10 subjects. The first bar shows the average overlap over the entire brain while the second bar shows the overlap only in the white matter regions (FA $>0.3$ ). Image shows OVL map for one of the subject. The red areas indicate high OVL. The main WM fiber tracts show very high OVL after registration using DROID. [Color figure can be viewed in the online issue, which is available at www.interscience.wiley.com]

of 1.0. We did not use the orientation features in DROID, in order for the method to be comparable for FA-based registration of Demons. Two subjects were registered to a template. We had two raters pick up 10 corresponding landmarks from each subject and template. These landmarks were chosen from the major and minor WM fibers that served to evaluate the matching accuracy.

C.2.3. Comparison with Other Deformable Registration Algorithms. Finally, we compared our registration method using 10 normal subjects with a deformable registration technique FNIRT applied to the FA maps and DTITK applied to the tensors directly (http://groups.google.com/group/dtitk) (Zhang et al., 2006). For FNIRT registration, a cubic spline function was chosen and the registration was carried out at four resolution iterations with 4,2,1,1 scheme. When comparing DROID with FNIRT only the intensity features were used while for comparison with DTITK, intensity as well as orientation descriptors were used. All the subjects were registered to a standard template (Wakana et al., 2004). DTITK was initialized using rigid registration followed by an affine registration and finally a nonlinear piecewise affine registration with optimized settings. A variance map over average FA was computed all the registration methods.

C.2.4. Creating Brain Atlas. In this section, we demonstrate the accuracy and applicability of our algorithm. We applied DROID to five mouse datasets and 30 adult human datasets. The group-averaged image was computed by voxel-wise averaging the corresponding tensors in the individual warped subjects using log-Euclidean averaging (Pennec et al., 2006).

\section{RESULTS}

A. Evaluation on Simulated Datasets. Figure 1 displays results for the registration performed on simulated datasets. One of the simulated datasets' FA is shown in Figure 1a. Figure 1b is the FA of the deformed image after registration using DROID. Figure

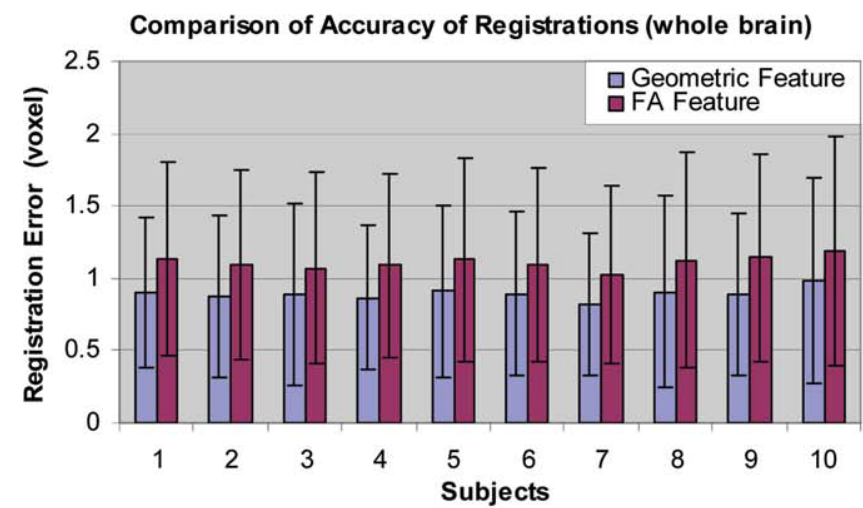

(a)

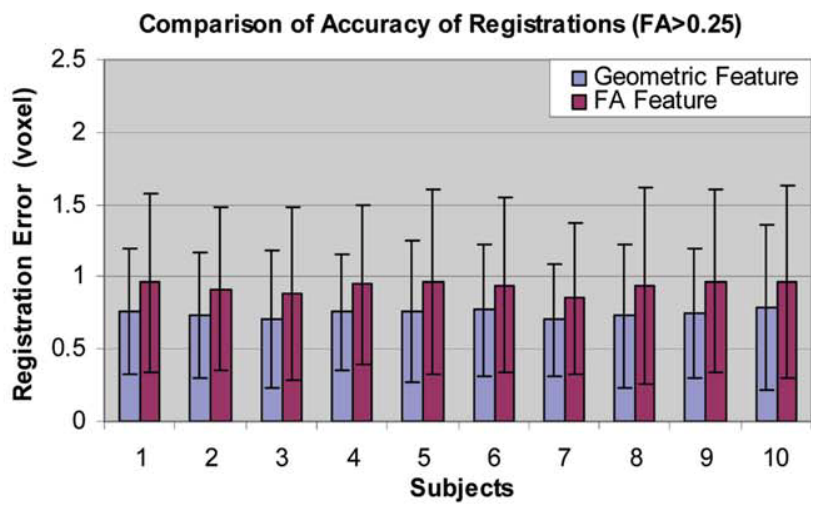

(b)

Figure 3. Comparison of the registration accuracy between DROID using intensity features and FA-based registration. (a) Shows the registration error computed from the whole brain, and (b) shows the registration error computed from WM fiber tracts with FA $>0.25$. It can be seen that DROID (when only the intensity based features are used) performs much better than registration based on FA features. [Color figure can be viewed in the online issue, which is available at www.interscience.wiley.com] 


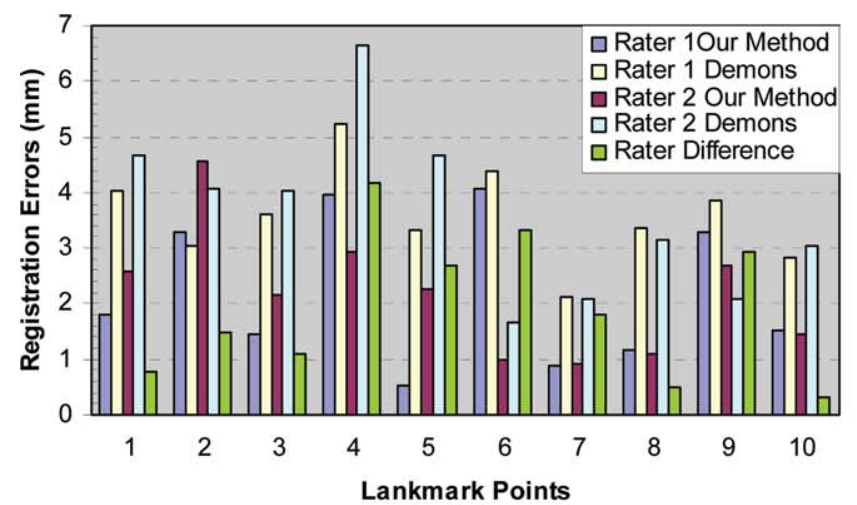

(a)

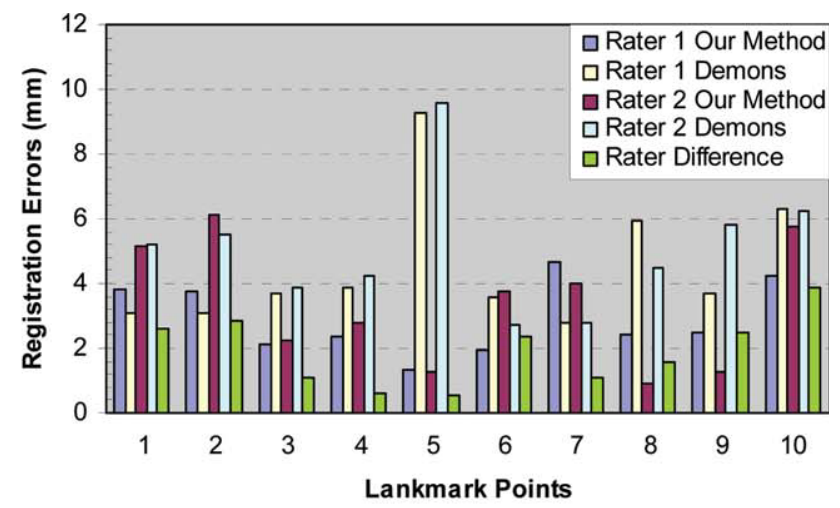

(b)

Figure 4. Comparison of the registration errors on each of 10 landmark points identified by two raters on both major and minor WM fiber tracts, in two subjects (a) and (b), respectively. [Color figure can be viewed in the online issue, which is available at www.interscience.wiley.com]

1c shows the FA of the template image. The plot in Figure 2 displays the tensor overlap for the 10 simulated datasets after the registration is carried out. The first bar shows the average OVL over the entire brain (average $0.8277 \pm 0.007$ ) while the second bar displays the OVL in the WM regions (FA $>0.3$ ). The overlap is high in the
WM regions with an average of $0.925 \pm 0.005$. Image in Figure 2 shows the OVL map. The red areas indicate the regions with very high overlap. It can be observed that all the major WM regions (e.g., the corpus callosum, the internal and the external capsule) have OVL values close to one indicating a high-quality matching.
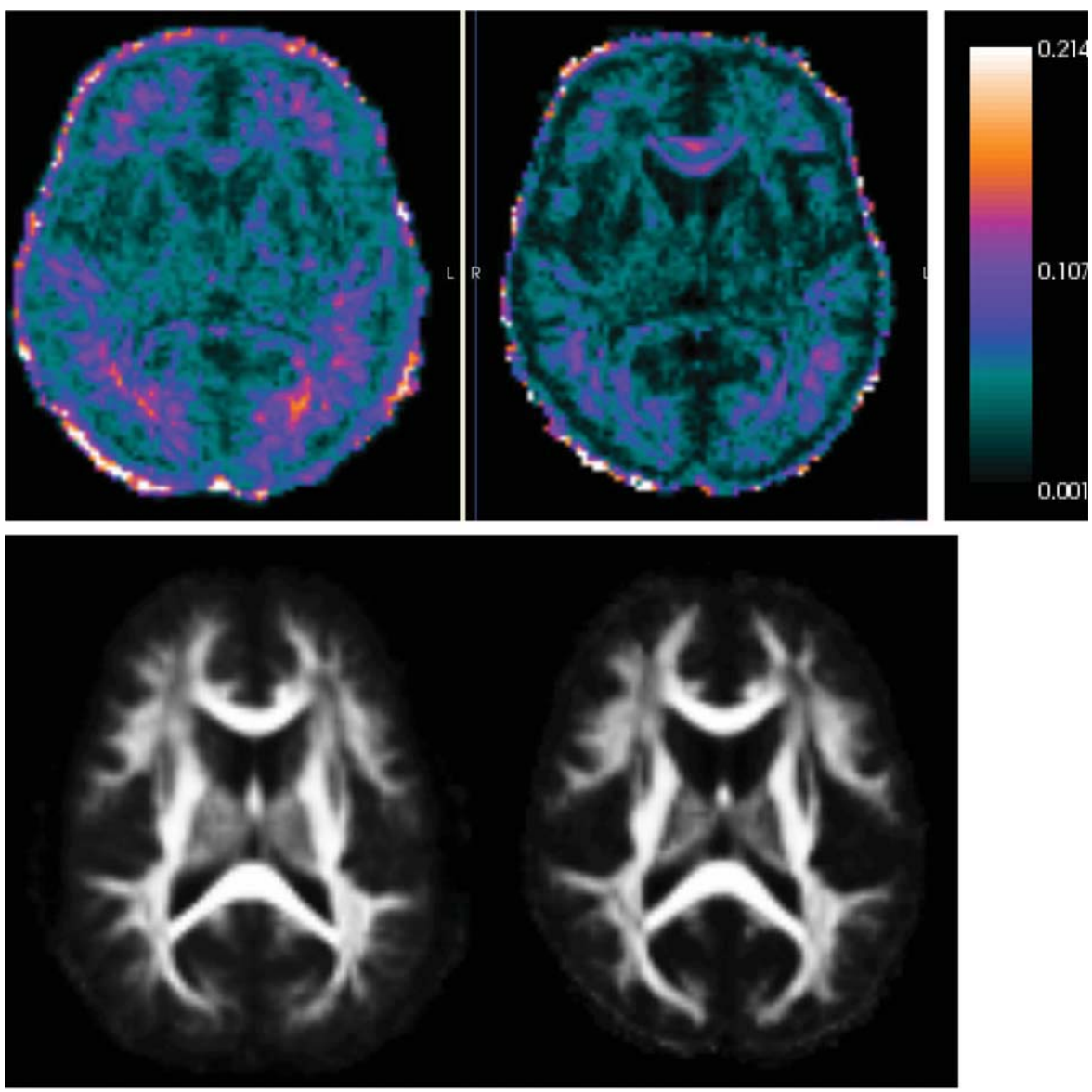

(a)

(b)

Figure 5. Comparison of registration using DROID and FNIRT. Top row shows the voxel-wise variance maps of the FA maps obtained by registering 10 subjects to a template using (a) FNIRT and (b) DROID. Inspection of the color-coded images show greater over all variance in FNIRT is higher, indicating high variability. Row 2 shows the mean FA maps. Again the mean FA obtained using DROID is sharper indicating lower anatomical variability. [Color figure can be viewed in the online issue, which is available at www.interscience.wiley.com] 


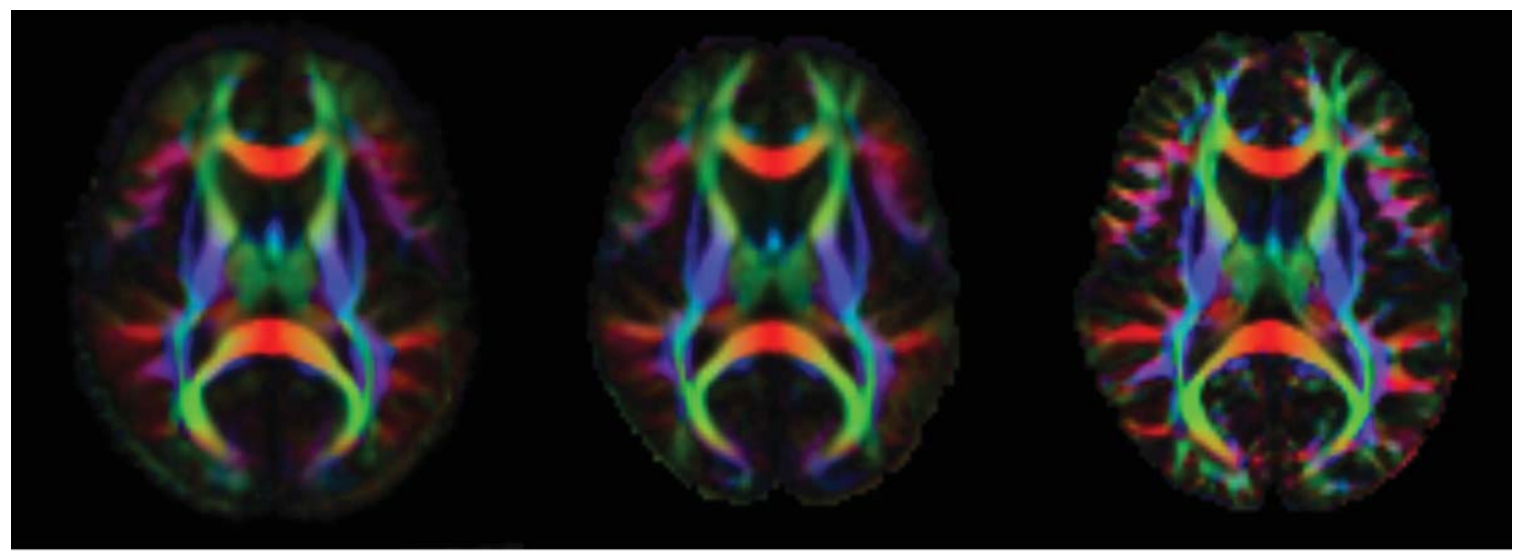

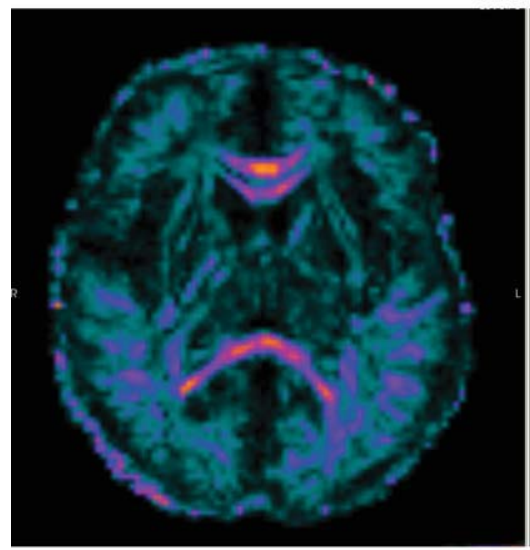

(a)

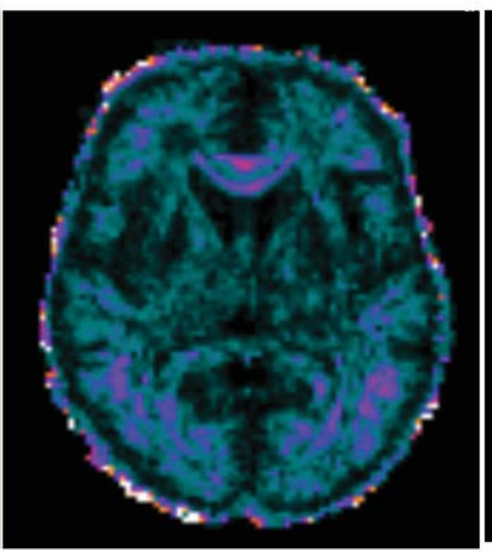

(b)

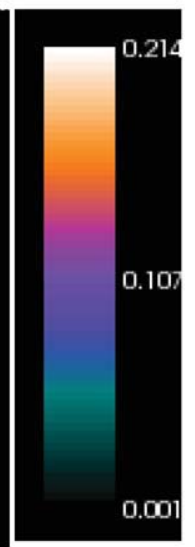

0.001

Figure 6. Comparison of DROID against DTITK. On the first line, the mean tensors computed from the 10 deformed subjects are shown while on the second line the variance over FA is illustrated. (a) DTITK (b) DROID, and (c) standard template. [Color figure can be viewed in the online issue, which is available at www.interscience.wiley.com]

Figure 3 shows the average registration error and the variance for each subject computed from the whole brain and WM fiber tracts, respectively. It demonstrates that using DROID with intensity features yields more accurate registration than using just the FA-based feature, with respective population means as 0.89 voxels and 1.11 voxels for the whole brain. Comparing Figures $3 \mathrm{a}$ and $3 \mathrm{~b}$ shows the registration to be superior in the WM fiber tracts. The respective population means using geometric feature and FA feature are 0.75 voxels and 0.93 voxels in the regions with $\mathrm{FA}>0.25$.

\section{B. Validation Based on Expert Identified Point}

Matches. For each pair of corresponding landmarks, we computed the registration error (the Euclidean norm between the point identified by the algorithm as a possible match, against the one identified by the rater) for our proposed method and the Demons algorithm. These results are shown in Figure 4. We also show the variation between two raters as the "rater difference" in Figure 4 for better understanding. From the plot it can be observed that average error using DROID is much lower than the demons algorithm. Specifically, at landmark point 4 in Figure $4 \mathrm{a}$ and at landmark point 5 in Figure $4 b$ the difference is extremely high.

\section{Comparison with Other Deformable} Registrations. Figure 5 shows the mean and variance of the reg- istered images using DROID and FNIRT. Visual inspection suggests that the mean FA map from DROID is sharper than the one computed from FNIRT. This implies that local anatomical nonlinear variability is reduced when DROID is used. The voxel-wise variance maps obtained from the 10 subjects indicates higher overall variance when FNIRT is used than when DROID is employed.

Figure 6 displays the mean color map and the variance computed from DTITK and DROID juxtaposed with the template tensor. Visual inspection suggests that DROID shows improved results than DTITK when compared with the template image. The OVL between the mean tensor and template confirms the results. (DROID OVL: $0.833 \pm 0.163$ while DTITK OVL: $0.828 \pm 0.180$ ).

D. Creating Brain Atlas. Figure 7 shows representative slices from the average images. These images are generated by color coding the principle eigenvector image weighted by the corresponding FA value. The edge map (WM boundary) is overlaid on the average for an effortless inspection. The same is repeated for human brains as is demonstrated in the second row of Figure 7.

\section{DISCUSSION}

We have developed a feature-based registration algorithm for DTI data that incorporates both intensity and orientation information available from the tensor. Through a sequence of experiments on 

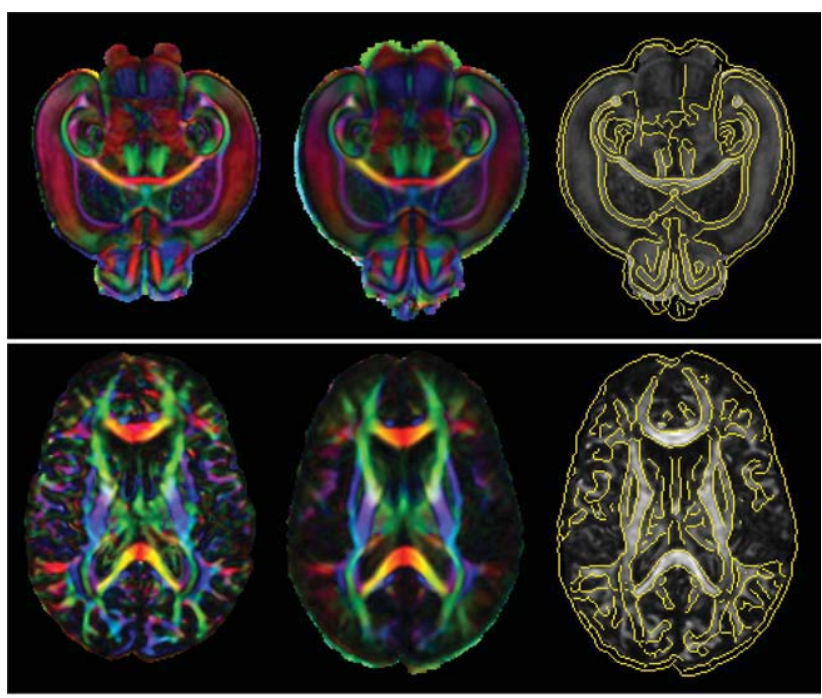

(a)

(b)

(c)

Figure 7. Spatial normalization of five mouse brains (row 1) and 30 human brains (row 2). (a) Color map of the template. (b) Color map of the group averaged image. (c) Edges extracted from FA map of group averaged image superimposed on the FA map of template. [Color figure can be viewed in the online issue, which is available at www.interscience.wiley.com]

real and simulated data we have established the applicability and accuracy of the registration algorithm.

Our algorithm includes various geometric features as compared to using just FA-based features. Distinctive attribute vectors, as are required for a good registration, can determine high similarity points close to them, but not with the rest of the image. These intensity features that combine anisotropy and diffusivity information take into consideration the neighborhood and give very specific match compared to using only FA. Similar to the geometric features, it is important to include the orientation descriptors for tensor images. Our registration method thus involves a combination of geometric and orientation features that can minimize the overall shape change as well as the differences in the WM fiber tracts.

As an initial step, the robustness of the algorithm was validated in itself by applying it to simulated datasets. One of the simulated datasets is shown in Figure 1. The overlap of the tensors with the template was evaluated over 10 subjects (Fig. 2). It was observed that the overlap was very high especially in the WM regions (FA > 0.3 ). This was confirmed from the color map in Figure $2 b$ where all the regions that are red have very high overlap (close to one). Figure 3 presented the overall registration accuracy of DROID (on the simulated datasets) in the whole brain and in the WM alone. This was compared against the registration achieved by an FA-based registration. The overall registration error was found to be low indicating a better registration.

In Figure 4, we evaluated the matching accuracy of the registration against point matches identified by human raters. Figure 4 illustrates that overall our method achieved more accurate and robust registration than the Demons algorithm did on the points in which the inter-rater variability was low. This suggests that DROID is able to register points that can be consistently matched by humans.

Next, we compared DROID with other methods that involve only FA registration (FNIRT) as well tensor registration methods like DTITK. For comparison with the FA-based registration, only intensity features were considered while for comparison against DTITK both intensity and orientation features were used. From Figure 5 it was observed that DROID performed better not only in the WM, but also in the GM, based on overall low variance in Figure $5 \mathrm{~b}$, top row. In addition, the mean was also sharper on using DROID, showing that the registration using DROID produces a better overlay and hence a sharper mean, indicating a low anatomical variability.

The visual inspection of the means and FA variance from Figure 6, suggests that DROID produced superior results when compared to DTITK. The average OVL using DROID was 0.833 as compared to 0.828 from DTITK. Finally, we applied our algorithm not only to human brain but also to mouse brains for testing the versatility of the algorithm by creating a template. In Figure 7 , we see the result of registering mouse and human data to a template. The registered tensor data is then averaged. The sharpness of the average in Figure $7 \mathrm{~b}$ as compared to the template in Figure $7 \mathrm{a}$, as well as the good matching of the overlaid edge map of the template on to the underlying average FA image of the registered subjects in Figure 7c, showed that a good spatial normalization was achieved and even the thin tracts like internal and external capsules were aligned well. Being able to create good averages is a prerequisite to doing statistical analysis on these brains, as the averaged brains form an atlas.

Advantage of using DROID is that it is very easy to use and takes less computation time. If a quick registration is required, one can switch off the orientation features and can still get a good nonlinear registration than using scalar images. Also, DROID works on most of the datasets (human, mouse, etc.) and it can be applied to any unit of diffusivity. Thus, the article provided a deformable registration algorithm for tensors that has been tested on datasets of various resolutions. The registration package is made available on our website (www.rad.upenn.edu/sbia/software).

\section{REFERENCES}

C. Pierpaoli, P. Jezzard, P.J. Basser, A. Barnett, and G.D. Chiro, Diffusion tensor MR imaging of human brain, Radiology 201 (1996), 637-648.

D.C. Alexander, C. Pierpaoli, P.J. Basser, and J.C. Gee, Spatial transformations of diffusion tensor magnetic resonance images, IEEE Trans Med Imaging 20 (2001), 1131-1139.

D. Xu, S. Mori, D. Shen, and C. Davatzikos, An approach to tensor fields reorientation based on optimized neighborhood, Hum Brain Mapp 50 (2003), $175-182$.

J. Yang, D. Shen, C. Davatzikos, and R. Verma, Diffusion tensor image registration using tensor geometry and orientation features, Proceedings of MICCAI, 2008, pp. 905-913.

H. Zhang, P.A. Yushkevich, D.C. Alexander, and J.C. Gee, Deformable registration of diffusion tensor MR images with explicit orientation optimization, Med Image Anal 10 (2006), 764-785.

A. Guimond, C.R.G. Guttmann, S.K. Warfield, and C.-F. Westin, Deformable registration of DT-MRI data based on transformation invariant tensor characteristics, Proceedings of ISBI, 2002, pp. 761-765.

Y. Cao, M. Miller, S. Mori, R.L. Winslow, and L. Younes, Diffeomorphic matching of diffusion tensor images, Proceedings of ISBI (2006): Proceedings of MMBIA p. 65.

B.T.T. Yeo, T. Vercauteren, P. Fillard, X. Pennec, P. Golland, N. Ayache, and O. Clatz, DTI registration with exact finite-strain differential, 5th IEEE International Symposium on Biomedical Imaging: From Nano to Macro, 2008, pp. 700-703. 
D.C. Alexander and J.C. Gee, Elastic matching of diffusion tensor images, Comput Vis Image Underst 77 (1999), 233-250.

D. Shen and C. Davatzikos, Hammer: Hierarchical attribute matching mechanism for elastic registration, IEEE Trans Med Imaging 21 (2002), 1421-1439.

R. Verma and C. Davatzikos, Matching of diffusion tensor images using gabor features, Proceedings of the IEEE International Symposium on Biomedical Imaging (ISBI), 2004, pp. 396-399.

U. Ziyan, M.R. Sabuncu, L.J. O’Donnell, and C.-F. Westin, Nonlinear registration of diffusion MR images based on fiber bundles, Proceedings of MICCAI, 2007, 351-358.

C.-F. Westin and S. Maier, Processing and visualization of diffusion tensor MRI, Med Image Anal 6 (2002), 93-108.

J. Canny, A computational approach to edge detection, IEEE Trans Pattern Anal Mach Intell 8 (1986), 679-698.

$\mathrm{X}$. Huang and Y. Sun, Hybrid image registration based on configural matching of scale-invariant salient region features, IEEE workshop on image and video registration, 2004.

M. Jenkinson and S. Smith, A global optimisation method for robust affine registration of brain images, Med Image Anal 5 (2001), 143-156.
D. Shen, Image registration by local histogram matching, Pattern Recogn 40 (2007), 1161-1171.

G.E. Christensen and H.J. Johnson, Consistent image registration, IEEE Trans Med Imag 20 (2001), 568-582.

Z. Xue, D. Shen, B. Karacali, J. Stern, D. Rottenberg, and C. Davatzikos, Simulating deformations of MR brain images for validation of atlas-based segmentation and registration algorithms, NeuroImage 33 (2006), 855-866.

S.M. Smith, M. Jenkinson, M.W. Woolrich, C.F. Beckmann, T.E.J. Behrens, H. Johansen-Berg, P.R. Bannister, M.D. Luca, I. Drobnjak, D.E. Flitney, R. Niazy, J. Saunders, J. Vickers, Y. Zhang, N.D. Stefano, J.M. Brady, and P.M. Matthews, Advances in functional and structural MR image analysis and implementation as FSL, Neuroimage 23 (2004), 208-219.

P. Basser and S. Pajevic, Statistical artifacts in diffusion tensor MRI caused by background noise, Magn Reson Med 44 (2000), 41-50.

S. Wakana, H. Jiang, L.M. Nagae-Poetscher, P.C.M. van Zijl, and S. Mori, Fiber tract-based atlas of human white matter anatomy, Radiology 230 (2004), 77-87.

X. Pennec, P. Fillard and N. Ayache, A Riemannian framework for tensor computing, Int J Comput Vis 66 (2006), 41-66. 